Research Article

\title{
Common Fixed-Point Theorems in Modular Function Spaces Endowed with Reflexive Digraph
}

\author{
Jaauad Jeddi $\mathbb{D},{ }^{1}$ Mustapha Kabil, ${ }^{1}$ and Samih Lazaiz $\mathbb{D}^{2}$ \\ ${ }^{1}$ Laboratory of Mathematics and Applications, Faculty of Sciences and Technologies Mohammedia, University Hassan II, \\ Casablanca, Morocco \\ ${ }^{2}$ LASMA Laboratory Department of Mathematics Faculty of Sciences, Dhar El Mahraz University Sidi Mohamed Ben Abdellah, \\ Fes, Morocco \\ Correspondence should be addressed to Jaauad Jeddi; jaauadjeddi@gmail.com
}

Received 11 April 2020; Accepted 17 July 2020; Published 3 August 2020

Academic Editor: Frédéric Mynard

Copyright (c) 2020 Jaauad Jeddi et al. This is an open access article distributed under the Creative Commons Attribution License, which permits unrestricted use, distribution, and reproduction in any medium, provided the original work is properly cited.

\begin{abstract}
The purpose of this work is to extend the Knaster-Tarski fixed-point theorem to the wider field of reflexive digraph. We give also a DeMarr-type common fixed-point theorem in this context. We then explore some interesting applications of the obtained results in modular function spaces.
\end{abstract}

\section{Introduction}

The study of fixed point results in partially ordered sets finds its root in the work of Knaster [1] and Tarski [2]. In 1955, Tarski published his work in the context of complete lattices; the result states that each monotone mapping from a complete lattice to itself has a fixed point. In [3], Abian and Brown extended the result of Knaster-Tarski to chaincomplete poset with a least or largest element and showed that every order-preserving map has a fixed point.

On the contrary, several fixed-point theorems in metric spaces endowed with a partial order have been stated and studied. In 2004, Ran and Reuring (see [4]) combined successfully the Banach Contraction Principle and Knaster-Tarski fixed point. They managed to prove that every monotone mapping in a complete metric space has a fixed point provided that it satisfies contraction condition only for comparable elements. Jachymski [5] managed to prove an equivalent result of Ran and Reuring's in a metric space endowed with a graph.

In the same vein, the authors in $[6,7]$ extended the result of Ran and Reuring to the case of monotone nonexpansive mappings. Their starting point was to approach the fixed point by iterative techniques and successive approximations. Recently, Espínola and Wiśnicki [8] generalized the above results in Hausdorff topological spaces endowed with partial order. The key ingredient in such a generalization is the compactness of the order intervals mixed with Knaster-Tarski fixed point. For more details, see $[9,10]$.

In this work, we generalize several known results in the context of topological spaces endowed with a digraph instead of partial order. For this purpose, we introduce the concept of $G$-regular monotone mapping and we give some applications in modular function spaces of the obtained results.

\section{Main Results}

Since the main result of this work relates topological properties to graphs, the following definition is needed. The interested reader can consult [11], for more details.

Definition 1. A directed graph or digraph $G$ is determined by a nonempty set $V(G)$ of its vertices and the set $E(G) \subset V(G) \times V(G)$ of its directed edges. A digraph is reflexive if each vertex has a loop. Given a digraph $G=(V, E)$,

(i) If whenever $(x, y) \in E(G) \Rightarrow(y, x) \notin E(G)$, then the digraph $G$ is called an oriented graph. 
(ii) A digraph $\mathrm{G}$ is transitive whenever $[(x, y) \in E(G)]$ and $[(y, z) \in E(G)] \Rightarrow(x, z) \in E(G)$, for any $x, y, z \in V(G)$.

(iii) A dipath of $G$ is a sequence $a_{0}, a_{1}, \ldots a_{n}, \ldots$ with $\left(a_{i}, a_{i+1}\right) \in E(G)$ for each $i \in \mathbb{N}$.

(iv) A finite dipath of length $n$ from $x$ to $y$ is a sequence of $n+1$ vertices $\left(a_{0}, a_{1}, \ldots, a_{n}\right)$ with $\left(a_{i}, a_{i+1}\right) \in$ $E(G)$ and $x=a_{0}$ and $y=a_{n}$.

(v) A closed directed path of length $n>1$ from $x$ to $y$, i.e., $x=y$, is called a directed cycle.

(v) A digraph is connected if there is a finite (di) path joining any two of its vertices, and it is weakly connected if $\widetilde{G}$ is connected.

(vi) $[x]_{G}$ is the set of all vertices which are contained in some path beginning at $x$, i.e., $y \in[x]_{G} \Leftrightarrow$ there exist $\left(a_{0}, a_{1}, \ldots, a_{n}\right)$ with $\left(a_{i}, a_{i+1}\right) \in E(G)$ and $x=a_{0}$.

We extend the notions of upper and lower bound and supremum and infimum known in the case of ordered sets to graph structures.

Definition 2. Let $G=(V(G), E(G))$ be a reflexive digraph and $a, b \in V(G)$.

(i) We define the $G$-intervals as follows:

$$
\begin{aligned}
{[a, \longrightarrow) } & =\left\{x \in V(G), x \in[a]_{G}\right\}, \\
(\longleftarrow, a] & =\left\{x \in V(G), a \in[x]_{G}\right\},
\end{aligned}
$$

and

$$
[a, b]=[a, \longrightarrow) \cap(\longleftarrow, b]
$$

(ii) For a subset $A$ of $V(G)$, we say that $a \in V(G)$ is a $G$-upper bound of $A$ if $a \in[x, \longrightarrow), \forall x \in A$ $G$-lower bound of $A$ if $a \in(\longleftarrow, x], \forall x \in A$

(iii) A $G$-upper bound of $A$ that belongs to $A$ is called $G$-maximal element of $A$, and a $G$-lower bound of $A$ that belongs to $A$ is called $G$-minimal element of A

(iv) We say that $a \in V(G)$ is a $G$-supremum of $A$ if

$a$ is a $G$-upper bound

For every $G$-upper bound $b$ of $A, a \in(\longleftarrow, b]$

(v) We say that $a \in V(G)$ is a $G$-infimum of $A$ if

$a$ is a G-lower bound and

For every G-lower bound $b$ of $A, a \in[b, \longrightarrow)$

The following example illustrates this last definition.

\section{Example 1}

(1) Let $V(G)=\mathbb{R}$ and $E(G)=\{(x, y)$ : $|x| \leq|y|\}$. $G$ is a reflexive digraph. Set $A_{1}=(-\infty, 0]$.

$a \in A_{1}$ is a $G$-upper bound if for each $x \in A_{1}$

$$
a \in[x, \longrightarrow) \Leftrightarrow|x| \leq|a|
$$

thus, $A_{1}$ has no $G$-upper bound.

$a \in A_{1}$ is a $G$-lower bound if for each $x \in A_{1}$

$$
a \in(\longleftarrow, x] \Leftrightarrow|a| \leq|x|
$$

thus, 0 is the only $G$-lower bound of $A_{1}$. Moreover, since $0 \in A_{1}$ it is the only $G$-minimal element and it is the $G$-infimum.

Since $A_{1}$ has no $G$-upper bound the sets of $G$-maximal and $G$-supremum are empty.

(2) Unlike the case of partially order, the $G$-supremum of set may be not unique. Indeed, we consider $A_{2}=[0,1]$, thus we have

The set of $G$-upper bound of $A_{2}$ is $(-\infty,-1] \cup[1, \infty)$

1 is the only $G$-maximal element of $A_{2}$

$a$ is $G$-supremum of $A_{2}$ if and only if $a \in\{-1,1\}$

Recall that a collection of sets $\mathscr{E}$ has the finite intersection property (f.i.p.), if, for every family $\mathscr{F}$ of members of $\mathscr{E}$, the intersection of $\mathscr{F}$ is nonempty provided that the intersection of all finite subfamilies of $\mathscr{F}$ are nonempty.

Definition 3. Let $G=(V(G), E(G))$ be a reflexive digraph; a subset $L$ of $V(G)$ is said $G$-directed, if every finite subset of $L$ has a $G$-upper bound in $L$.

We then get the following generalization of the result obtained in [8] for graphs.

Lemma 1. Let $G=(V(G), E(G))$ be a reflexive digraph; if $G$ has the finite intersection property for $G$-intervals, then every $G$-directed subset $L$ of $V(G)$ has a G-supremum.

Proof 1 . We consider the set $M=\cap_{x \in L}[x, \longrightarrow)$, as $L$ is $G$-directed; every finite intersection $\cap_{i=1}^{n}\left[x_{i}, \longrightarrow\right)$, where $x_{1}, x_{2}, \ldots x_{n} \in L$, is nonempty since it contains every $G$-upper bound that is in $L$ of the finite subset $\left\{x_{1}, x_{2}, \ldots x_{n}\right\}$ and as $G$ has the finite intersection property, and $M$ is nonempty.

Let us consider now the set $M^{\prime}=\cap_{x \in L, y \in M}[x, y]$, then, again, every finite intersection $\bigcap_{i=1}^{n}\left[x_{i}, y_{i}\right]$, where $x_{1}, x_{2}, \ldots x_{n} \in L$ and $y_{1}, y_{2}, \ldots y_{n} \in M$ is nonempty (also it contains any $G$-upper bound that is in $L$ of the finite subset $\left.\left\{x_{1}, x_{2}, \ldots x_{n}\right\}\right)$ and $G$ has the finite intersection property $M^{\prime}$ which is nonempty. And, it is clear that every element of $M^{\prime}$ is a $G$-supremum of $L$.

Recall that a map $T: X \longrightarrow X$ is said to be $G$-monotone if for all $x, y \in X$, whenever $y \in[x]_{G}$, then $T(y) \in[T(x)]_{G}$. Next, we introduce the notion of $G$-regular monotone.

Definition 4. Let $X$ be a set endowed with a graph $G=$ $(V(G), E(G))$ a map $T: X \longrightarrow X$ is said to be $G$-regular monotone; if $T$ is $G$-monotone and for every $x, y \in X$, if $x \in[y]_{G}$ and $y \in[x]_{G}$, then $T(x)=T(y)$. 

follows.

The following theorem is the cornerstone of what

Theorem 1. Let $X$ be a topological space endowed with a reflexive digraph $G=(V(G), E(G))$ such that $G$-intervals are compact, and let $T: X \longrightarrow X$ be G-regular monotone map; if there exists $x_{0} \in X$ such that $T\left(x_{0}\right) \in\left[x_{0}\right]_{G}$, then the set of fixed points of $T$ is not empty and has a G-maximal element.

Proof. Let $L=\left\{T^{n}\left(x_{0}\right): n \in \mathbb{N}\right\}$. Then, $L$ is $G$-directed and for all $x \in L, T(x) \in L$ and $T(x) \in[x]_{G}$. Set now

$$
\begin{aligned}
\mathscr{F}= & \{\mathrm{JG}-\text { directed } L \subset J, \text { and } \forall x \in J, T(x) \in \\
& \left.J \text { and } T(x) \in[x]_{G}\right\},
\end{aligned}
$$

then $\mathscr{F}$ is nonempty inductive set with respect to the $c$ order (if $\left(J_{i}\right)_{i \in I}$ is a chain in $\mathscr{F}$ then $\cup_{i \in I} J_{i}$ is an upper bound of $\left(J_{i}\right)_{i \in I}$ in $\left.\mathscr{F}\right)$.

By Zorn's lemma, there exists a maximal $G$-directed set $L_{0}$ such that $L \subset L_{0}$ and for all $x \in L_{0}, T(x) \in L_{0}$, and $T(x) \in[x]_{G}$. Since the $G$-intervals are compacts, $G$ has the finite intersection property for $G$-intervals; thus, $L_{0}$ has a $G$-supremum $s$.

Now, for all $x \in L_{0}$, we have $s \in[x]_{G}$ and $T(s) \in[T(x)]_{G}$ and $T(x) \in[x]_{G}$ for all $x \in L_{0}$. Hence, $T(s)$ is a $G$-upper bound of $L_{0}$, that is, $T(s) \in[s]_{G}$. We then claim that $s \in L_{0}$. Indeed if $s \notin L_{0}$, then

$$
\widetilde{L}=L_{0} \cup\left\{T^{n}(s), n \in \mathbb{N}\right\},
$$

is a $G$-directed subset of $X$ that contains strictly $L_{0}$ (and thus $J$ as well) and such that, for all $x \in \widetilde{L}, T(x) \in \widetilde{L}$ and $T(x) \in[x]_{G}$, which is in contradiction with maximality of $L_{0}$.

As $s \in L_{0}$ and $T(s) \in L_{0}$ too, we have $s \in[T(s)]_{G}$ and as $T(s) \in[s]_{G}$; we get then $T(T(s))=T(s)$, that is, $T(s)$ is a fixed point of $T$.

Notice that if $z$ is a fixed point of $T, z \in L_{0}$, and thus $s \in[z]_{G}$ and $T(s) \in[T(z)]$ as $T$ is $G$-monotone.

Example 2. Let $X$ the unit disc in $\mathbb{C}$, i.e., $X=$ $\{z \in \mathbb{C}, \quad|z|=1\}$, endowed with the graph $G=(V(G)$, $E(G))$, where $V(G)=X$ and $(x, y) \in E(G)$ iff $|x| \leq|y|$.

Consider $T: X \longrightarrow X$ defined $T(x)=|x|$ for all $x \in X$ with the usual topology.

It is easy then to check that $G$-intervals are compact, and that $T$ is $G$-regular monotone mapping and that 1 is a fixed point for $T$ (every real positive number in $X$ is indeed a fixed point for $T$ ).

In the same way, we obtain a common fixed point for commuting family of $G$-monotone mappings.

Theorem 2. Let $X$ be a topological space endowed with a reflexive digraph $G=(V(G), E(G))$ such that $G$-intervals are compact, and let $\left\{T_{\lambda}: X \longrightarrow X, \lambda \in \Lambda\right\}$ be a family of commuting G-regular monotone mappings; if there exists $x_{0} \in X$ such that $T_{\lambda}\left(x_{0}\right) \in\left[x_{0}\right]_{G}$ for all $\lambda \in \Lambda$ then the set of common fixed points of the family $\left(T_{\lambda}\right)_{\lambda \in \Lambda}$ is nonempty and has a G-maximal element.

Proof. Let

$L=\left\{T_{\lambda_{1}} \cdot T_{\lambda_{2}} \cdot \ldots \cdot T_{\lambda_{n}}\left(x_{0}\right): n \in \mathbb{N}\right.$ and $\left.\lambda_{1} \ldots \lambda_{n} \in \Lambda\right\} ;$ then, $L$ is $G$-directed and we have $T_{\lambda}(x) \in L$ and $T_{\lambda}(x) \in[x]_{G}$ for all $x \in L$ and $\lambda \in \Lambda$. Let

$$
\begin{aligned}
\mathscr{F}= & \{\mathrm{JG}-\text { directed } L \subset J, \text { and } \forall x \in J, \forall \lambda \in \Lambda, \\
& \left.T_{\lambda}(x) \in J \text { and } T_{\lambda}(x) \in[x]_{G}\right\} .
\end{aligned}
$$

Then, $\mathscr{F}$ is a nonempty inductive set with respect to the inclusion order. Indeed, if $\left(J_{i}\right)_{i \in I}$ is a chain in $\mathscr{F}$, then $\cup_{i \in I} J_{i}$ is an upper bound of $\left(J_{i}\right)_{i \in I}$ in $\mathscr{F}$. By Zorn's lemma, there exists a maximal $G$-directed set $L_{0}$ such that $L \subset L_{0}$, and for all $x \in L_{0}$ and $\lambda \in \Lambda$, we get $T_{\lambda}(x) \in L_{0}$ and $T_{\lambda}(x) \in[x]_{G}$. As $G$ has the finite intersection property for $G$-intervals, $L_{0}$ has a $G$-supremum $s$.

For all $x \in L_{0}$, we have $s \in[x]_{G}$ and $T_{\lambda}(s) \in\left[T_{\lambda}(x)\right]_{G}$ and $T_{\lambda}(x) \in[x]_{G}$ for all $x \in L_{0}$ and for all $\lambda \in \Lambda$. Hence, $T_{\lambda}(s)$ is a $G$-upper bound of $L_{0}$, for any $\lambda \in \Lambda$. That is, $T_{\lambda}(s) \in[s]_{G}, \quad \forall \lambda \in \Lambda$. We then claim that $s \in L_{0}$, indeed if $s \notin L_{0}$, then

$$
\widetilde{L}=L_{0} \cup\left\{T_{\lambda_{1}} \circ T_{\lambda_{2}} \circ \ldots \circ T_{\lambda_{n}}(s): n \in \mathbb{N} \text { and } \lambda_{1} \ldots \lambda_{n} \in \Lambda\right\},
$$

is a $G$-directed subset of $X$ that contains strictly $L_{0}$ (and thus $J$ as well) and such that $\forall x \in \widetilde{L}, \forall \lambda \in \Lambda, \quad T_{\lambda}(x) \in \widetilde{L}$, and $T_{\lambda}(x) \in[x]_{G}$, which is in contradiction with maximality of $L_{0}$.

Now again, as $s \in L_{0}$, then, for each $\lambda \in \Lambda, T_{\lambda}(s) \in L_{0}$ and $s \in\left[T_{\lambda}(s)\right]_{G}$; hence, $T_{\mu}\left(T_{\lambda}(s)\right)=T_{\mu}(s), \forall \lambda, \mu \in \Lambda$. By commutativity, $T_{\mu}(s)$ is a common fixed point for the family of mappings $\left(T_{\lambda}\right)_{\lambda \in \Lambda}$.

Finally, if $z$ is a common fixed point of the family of mappings $\left(T_{\lambda}\right)_{\lambda \in \Lambda}$ then $z \in L$ thus $s \in[z]_{G}$ and $T_{\mu}(s) \epsilon$ $\left[T_{\mu}(z)\right]_{G}$, for all $\mu \in \Lambda$. Then, $T_{\mu}(s)$ is $G$-maximal element of the set of common fixed points of $\left(T_{\lambda}\right)_{\lambda \in \Lambda}$.

\section{Application to Modular Function Spaces}

For the sake of completeness, we begin by recalling some definitions and properties of modular function spaces that we used later. For more details, see [12].

Let $\Omega$ be a nonempty set and $\mathscr{P}$ a nontrivial $\delta$-ring of subsets of $\Omega$, and let $\Sigma$ be the smallest $\sigma$-algebra of subsets of $\Omega$ such that $\Sigma$ contains $\mathscr{P}$ such that $E \cap A \in \mathscr{P}$ for every $E \in \mathscr{P}$ and $A \in \Sigma ; K_{n} \uparrow \Omega$, where $K_{n} \in \mathscr{P}$, for all $n$.

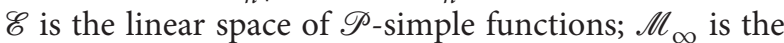
set of measurable functions. We denote by $1_{A}$ the characteristic function of $A$, where $A \subset \Omega$.

Definition 5. An even convex function $\rho: \mathscr{M}_{\infty} \longrightarrow[0,+\infty]$ is called regular convex function pseudomodular if

(i) $\rho(0)=0$.

(ii) $\rho$ is monotone, i.e., if for $f, g \in \mathscr{M}_{\infty}, f(\omega) \leq g(\omega)$ for all $\omega \in \Omega$, then $\rho(f) \leq \rho(g)$. 
(iii) $\rho$ is orthogonally subadditive, i.e., $\rho\left(f .1_{A \cup B}\right)=$ $\rho\left(f .1_{A}\right)+\rho\left(f .1_{B}\right)$, whenever $A, B \in \Sigma$ and $A \cap B=$ $\varnothing$ and $f \in \Sigma$.

(iv) $\rho$ has the Fatou property, i.e., if $\left(\left|f_{n}(\omega)\right|\right)_{n} \uparrow|f(\omega)|$, for all $\omega \in \Omega, f_{n}, f \in \mathscr{M}_{\infty}$ then $\rho\left(f_{n}\right) \uparrow \rho(f)$.

(v) $\rho$ is order continuous in $\mathscr{E}$, i.e., $g_{n} \in \mathscr{E}$ and $\left|g_{n}\right| \downarrow 0$ implies $\rho\left(g_{n}\right) \downarrow 0$.

Let $\rho$ be a regular convex function pseudomodular; we then introduce these notions:

(i) A set $A \in \Sigma$ is said $\rho$-null, if $\rho\left(g \cdot 1_{A}\right)=0, \forall g \in \mathscr{E}$.

(ii) A property $(P)$ is said to hold $\rho$ almost everywhere if the exceptional set is $\rho$-null.

(iii) We will identify pair of measurable sets whose symmetric difference is $\rho$-null, as well as pair of measurable function differing only on a $\rho$-null set.

(iv) $\mathscr{M}(\Omega, \Sigma, \mathscr{P}, \rho)=\left\{f \in \mathscr{M}_{\infty}:|f(\omega)|<\infty \rho-\right.$ a.e. $\}$ briefly noted $\mathscr{M}$.

(v) $\rho$ is said a regular convex function modular if $\rho(f)=0$ implies $f=0 \rho$-a.e.

(vi) We denote by $\mathfrak{R}$ the set of all nonzero regular convex function modulars on $\Omega$.

\section{Definition 6. Let $\rho \in \mathfrak{R}$ :}

(i) We say that $\left(f_{n}\right)_{n} \in L_{\rho} \rho$-converges to $f$, and write $f_{n} \longrightarrow f(\rho)$, if $\rho\left(f_{n}-f\right) \longrightarrow 0$, and a sequence $\left(f_{n}\right)_{n} \in L_{\rho}$ is called $\rho$-Cauchy if $\rho\left(f_{n}-f_{m}\right) \longrightarrow 0$ as $(n, m) \longrightarrow \infty$.

(ii) A set $B \subset L_{\rho}$ is called $\rho$-closed, if for any sequence $\left(f_{n}\right)_{n} \in B, f_{n} \longrightarrow f(\rho)$ implies $f \in B$.

(iii) A set $B \subset L_{\rho}$ is called $\rho$-bounded, if his diameter $\delta_{\rho}(B)=\sup \{(\rho(f-g) / f), g \in B\}$ is finite.

(iv) A set $B \subset L_{\rho}$ is called $\rho$-compact, if for any sequence $\left(f_{n}\right)_{n} \in L_{\rho}$ there exists a subsequence $\left(f_{k_{n}}\right)_{n}$ and $f \in B$ such that $\left(f_{k_{n}}\right) \rho$-converges to $f$.

(v) A set $B \subset L_{\rho}$ is called $\rho$-a.e.-compact, if for any sequence $\left(f_{n}\right)_{n} \in L_{\rho}$ there exists a subsequence $\left(f_{k_{n}}\right)_{n}$ and $f \in B$ such that $\left(f_{k_{n}}\right) \rho$-a.e.-converges to $f$.

(vi) A set $B \subset L_{\rho}$ is called $\rho$-a.e.-closed, if for any sequence $\left(f_{n}\right)_{n} \in B, f_{n} \longrightarrow f, \rho$-a.e. implies $f \in B$.

\section{Definition 7. Let $\rho \in \mathfrak{R}$.}

The modular function space is the vector space $L_{\rho}(\Omega, \Sigma)$ or briefly $L_{\rho}$ defined by

$$
L_{\rho}=\left\{f \in \mathscr{M} ; \lim _{\lambda \longrightarrow 0} \rho(\lambda . f)=0\right\} .
$$

The map $\left\|_{\rho}\right\|_{\rho}: L_{\rho} \longrightarrow[0,+\infty)$ is defined by

$$
\|f\|_{\rho}=\inf \left\{\alpha>0 ; \rho\left(\frac{f}{\alpha}\right) \leq 1\right\} \text {, }
$$

which is called norm of Luxembourg on $L_{\rho}$.
The following properties play a prominent role in the study of modular function spaces.

\section{Definition 8. Let $\rho \in \mathfrak{R}$ :}

(i) We say that $\rho$ has the $\Delta_{2}$-property, if $\rho\left(2 f_{n}\right) \longrightarrow 0$ whenever $\rho\left(f_{n}\right) \longrightarrow 0,\left(\left(f_{n}\right)_{n} \in L_{\rho}\right)$.

(ii) We say that $\rho$ has the $\Delta_{2}$-type condition, if there exists $k \in[0,+\infty)$ such that $\rho(2 f) \leq k \rho(f)$, for any $f \in L_{\rho}$.

We need the following definition of the growth function.

Definition 9 (see [12]). Let $\rho$ be a function modular; the function $\omega_{\rho}:[0,+\infty] \longrightarrow[0,+\infty]$ is defined by

$$
\omega_{\rho}(t)=\sup \left\{\frac{\rho(t f)}{\rho(f)}: f \in L_{\rho} \text { and } 0<\rho(f)<\infty\right\},
$$

which is called the growth of $\rho$.

The growth function has the following properties.

Proposition 1 (see [12]). Let $\rho \in \mathfrak{R}$ that has the $\Delta_{2}$-type condition, and $\omega_{\rho}$ its growth function; then,

(i) $\omega_{\rho}(t)<\infty, \forall t \in[0,+\infty[$.

(ii) $\omega_{\rho}:[0,+\infty[\longrightarrow[0,+\infty[$ is convex, and strictly increasing, it is then also continuous.

(iii) $\omega_{\rho}(\alpha \beta) \leq \omega_{\rho}(\alpha) \omega_{\rho}(\beta)$, for all $\alpha, \beta \in[0,+\infty)$.

(iv) $\omega_{\rho}^{-1}(\alpha) \omega_{\rho}^{-1}(\beta) \leq \omega_{\rho}^{-1}(\alpha \beta)$, for all $\alpha, \beta \in[0,+\infty)$, where $\omega_{\rho}^{-1}$ is the inverse function of $\omega_{\rho}$.

(v) $\|f\|_{\rho} \leq\left(1 / \omega_{\rho}^{-1}(1 / \rho(f))\right)$, for any $f \in L_{\rho}$.

Proofs of following theorems could be found in [12].

Theorem 3. Let $\rho \in \mathfrak{R}$ :

(i) $\left(L_{\rho},\|.\|_{\rho}\right)$ is a complete normed space, and $L \rho$ is $\rho$-complete.

(ii) $\left\|f_{n}\right\|_{\rho} \longrightarrow 0$ iff $\rho\left(\alpha f_{n}\right) \longrightarrow 0$ for every $\alpha>0$.

(iii) If $\rho\left(f_{n}-f\right) \longrightarrow 0$ there exists $\left(f_{n_{k}}\right)_{k}$ subsequence of $\left(f_{n}\right)_{n}$ such that $f_{n_{k}} \longrightarrow f, \rho$-a.e.

(iv) If $f_{n} \longrightarrow f \rho$-a.e, then $\rho(f) \leq \liminf _{n \longrightarrow+\infty} \rho\left(f_{n}\right)$ (the Fatou property).

(v) If $\rho$ has the $\Delta_{2}$-property and $\rho\left(\alpha f_{n}\right) \longrightarrow 0$ for $\alpha>0$, then $\left\|f_{n}\right\|_{\rho} \longrightarrow 0$.

A modular $\rho$ is said $\sigma$-finite if there exists an increasing sequence of sets $K_{n} \in \mathscr{P}$ such that, for every $n \in \mathbb{N}, 0<$ $\rho\left(K_{n}\right)<\infty$ and $\Omega=\cup_{n \in \mathbb{N}} K_{n}$.

Let $d: L_{\rho} \times L_{\rho} \longrightarrow[0,+\infty)$ defined by

$$
d(f, g)=\sum_{k=1}^{+\infty} \frac{1}{2^{k}} \frac{1}{\rho\left(1_{K_{k}}\right)} \rho\left(\frac{|f-g|}{1+|f-g|} 1_{K_{k}}\right) \text {. }
$$

Theorem 4. Let $\rho \in \Re$ if $\rho$ is $\sigma$-finite and has the $\Delta_{2}$-type condition; then, for any $f, g \in L_{\rho}$,

(1) $d(f, g)=0$ if $f f=g \rho$-a.e.

(2) $d(f, g)=d(g, f)$ 
(3) $d(f, g) \leq(\omega(2) / 2)(d(f, h))+d(h, g)$

and if $\left(f_{n}\right)_{n}$ is a sequence in $L_{\rho}$ that is $\rho$-a.e. convergent to $f$, then $\lim _{n \longrightarrow+\infty} d\left(f_{n}, f\right)=0$.

Moreover, if $\lim _{n \longrightarrow+\infty} d\left(f_{n}, f\right)=0$, then there exists a subsequence $\left(f_{n_{k}}\right)_{k}$ that converges $\rho$-a.e. to $f$.

Here is the first application of our main result to modular function spaces.

Theorem 5. Let $\rho \in \mathfrak{R}$, that has the $\Delta_{2}$-property and $G$ a digraph on $L_{\rho}$ such that $G$-intervals are $\rho$-compact. Let $T_{\lambda}: L_{\rho} \longrightarrow L_{\rho}, \lambda \in \Lambda$ be a family of commuting G-regular monotone mappings; if there exists $f_{0} \in L_{\rho}$ such that $T_{\lambda}\left(f_{0}\right) \in\left[f_{0}\right]_{G}, \forall \lambda \in \Lambda$, then the set of common fixed points of the family $\left(T_{\lambda}\right)_{\lambda \in \Lambda}$ is not empty and has a G-maximal element.

Proof. As $\rho$ has the $\Delta_{2}$-property, then the $\rho$-convergence is equivalent to convergence in the Banach space $\left(L_{\rho},\|.\|_{\rho}\right)$, which means that every $\rho$-compact subset of $L_{\rho}$ is a compact in $\left(L_{\rho},\|.\|_{\rho}\right)$; we can then apply Theorem 2 .

Requiring more conditions on the function modular $\rho$ one can suppose that $G$-intervals are only $\rho$-a.e. compact.

Theorem 6. Let $\rho \in \mathfrak{R}$, a $\sigma$-finite function modular, that has the $\Delta_{2}$-type condition and $G$ a digraph on $L_{\rho}$ such that $G$-intervals are $\rho$-a.e.-compact. Let $T_{\lambda}: L_{\rho} \longrightarrow L_{\rho}, \lambda \in \Lambda$ be a family of commuting G-regular monotone mappings; if there exists $f_{0} \in L_{\rho}$ such that $T_{\lambda}\left(f_{0}\right) \in\left[f_{0}\right]_{G}, \forall \lambda \in \Lambda$, then the set of common fixed points of the family $\left(T_{\lambda}\right)_{\lambda \in \Lambda}$ is not empty and has a G-maximal element.

Proof. Indeed, Theorem 4 states that $\left(L_{\rho}, d\right)$ is a b-metric space, and then sequential compactness is equivalent to compactness (the usual argument, which proves that fact for metric spaces, still holds in b-metric spaces). Now, if a subset $K$ of $L_{\rho}$ is $\rho$.a.e.-compact, then from every sequence of elements of $K$ one can extract a subsequence that converges $\rho$-a.e.; then, by Theorem 4 , one can extract a subsequence that converges in the b-metric space $\left(L_{\rho}, d\right)$. That is, $K$ is sequentially compact in $\left(L_{\rho}, d\right)$ and thus compact, which implies that $G$-intervals are compact for the topology of the b-metric space $\left(L_{\rho}, d\right)$, then using Theorem 2 we get the result.

\section{Data Availability}

No data were used to support this study.

\section{Conflicts of Interest}

The authors declare that there are no conflicts of interest regarding the publication of this paper.

\section{References}

[1] B. Knaster, "Un théorème sur les fonctions d'ensembles," Annals of the Polish Society of Mathematics, vol. 6, pp. 133-134, 1928.
[2] A. Tarski, "A lattice-theoretical fixpoint theorem and its applications," Pacific Journal of Mathematics, vol. 5, no. 2, pp. 285-309, 1955.

[3] S. Abian and A. B. Brown, "A theorem on partially ordered sets, with applications to fixed point theorems," Canadian Journal of Mathematics, vol. 13, pp. 78-82, 1961.

[4] A. C. M. Ran and M. C. B. Reurings, "A fixed point theorem in partially ordered sets and some applications to matrix equations," Proceedings of the American Mathematical Society, vol. 132, pp. 1435-1443, 2004.

[5] J. Jachymski, "The contraction principle for mappings on a metric space with a graph," Proceedings of the American Mathematical Society, vol. 136, no. 4, pp. 1359-1373, 2008.

[6] M. R. Alfuraidan and M. A. Khamsi, "A fixed point theorem for monotone asymptotically nonexpansive mappings," Proceedings of the American Mathematical Society, vol. 146, no. 6, pp. 2451-2456, 2016.

[7] M. Bachar and M. A. Khamsi, "Delay differential equation in metric spaces: a partial ordered sets approach," Fixed Point Theory and Applications, vol. 193, 2014.

[8] R. Espínola and A. Wiśnicki, "The Knaster-Tarski theorem versus monotone nonexpansive mappings," Bulletin of the Polish Academy of Sciences Mathematics, vol. 66, pp. 1-7, 2018.

[9] J. Jeddi, M. Kabil, and S. Lazaiz, "Some fixed point theorems in modular function spaces endowed with a graph," Abstract and Applied Analysis, vol. 2020, Article ID 2135859, 7 pages, 2020.

[10] M. A. Khamsi, "Notes on knaster-tarski theorem versus monotone nonexpansive mappings," Moroccan Journal of Pure and Applied Analysis, vol. 4, no. 1, pp. 1-8, 2018.

[11] M. Rigo, Advanced Graph Theory and Combinatorics, John Wiley \& Sons, Hoboken, NY, USA, 2016.

[12] M. A. Khamsi and W. M. Kozlowski, Fixed Point Theory in Modular Function Spaces, Birkhäuser, Basel, Switzerland, 2015. 